\title{
CARCINOMA INDIFERENCIADO CON CÉLULAS GIGANTES DE TIPO OSTEOCLASTOS EN LA CABEZA DE PÁNCREAS
}

\author{
UNDIFFERENTIATED CARCINOMA WITH OSTEOCLAST-LIKE GIANT CELLS (UC-OGC) \\ OF THE HEAD OF PANCREAS \\ Eugenio Palomino 1,a, Jesús E. Talavera $2,3, \mathrm{~b}$
}

\begin{abstract}
RESUMEN
El carcinoma indiferenciado con células indiferenciadas tipo osteoclasto (UC-OGC) en cabeza de páncreas, es extremadamente raro. Las características clínicopatológicas, de imagen y la apariencia topográfica no están bien clasificadas. Este reporte de caso describe un paciente con UC-OGC de cabeza de páncreas. Nuestro paciente un adulto mayor de 75 años de edad, presentaba un tiempo de enfermedad considerable, sin sintomatología relevante hasta las etapas avanzadas del tumor, debutando con molestias gástricas someras, luego pérdida de peso. En los exámenes diagnósticos hallaron la presencia de un tumor que comprometía el estómago, duodeno y páncreas. El diagnóstico definitivo lo dio el estudio anatomopatológico. No se dispone de datos clínicos suficientes para un tratamiento que garantice una mayor tasa de supervivencia en los pacientes, sin embargo, la pancreatoduodenectomía es actualmente la alternativa que mejores resultados ofrece, por otra parte, el pronóstico después de la cirugía y la tasa de recurrencia sigue incierto.
\end{abstract}

Palabras clave: Carcinoma de células gigantes; Carcinoma indiferenciado; Páncreas; Cabeza de páncreas. (fuente: DeCS BIREME)

\begin{abstract}
Undifferentiated carcinoma with undifferentiated osteoclast-like cells (UC-OGC) in the head of the pancreas is extremely rare. The clinicalpathological, imaging and topographic appearance characteristics are not well classified. This case report describes a patient with UC-OGC of the Pancreas Head. Our patient, an adult over 75 years of age, had a considerable illness time, without relevant symptoms until the advanced stages of the tumor, debuting with shallow gastric discomfort, then weight loss. In the diagnostic tests they found the presence of a tumor that compromised the stomach, duodenum and pancreas. The definitive diagnosis was given by the pathological study. There are not enough clinical data available for a treatment that guarantees a higher survival rate in patients, however, pancreatoduodenectomy is currently the alternative that offers the best results, on the other hand, the prognosis after surgery and the recurrence rate still uncertain.
\end{abstract}

Key words: Carcinoma; Giant Cell; undifferentiated carcinoma; Pancreas; Pancreas head. (source: MeSH NLM)

\section{INTRODUCCIÓN}

El carcinoma indiferenciado con células gigantes de tipo osteoclasto (UC-OGC) ubicado en la cabeza del páncreas es muy inusual existiendo pocos casos reportados hasta el momento. Forma parte de los tipos de tumores pancreáticos exocrinos menos comunes $^{1,2}$. Esta patología representa menos del $1 \%$ de todas las neoplasias malignas pancreáticas y fue descrita por primera vez por Rosai ${ }^{3,4}$. Se caracteriza por la presencia de dos poblaciones celulares: células epiteliales mononucleares polimórficas indiferenciadas y células gigantes multinucleadas benignas.

\footnotetext{
${ }^{1}$ Hospital Nacional Edgardo Rebagliati Martins, Lima-Perú.

${ }^{2}$ Instituto de Investigación en Ciencias Biomédicas, Universidad Ricardo Palma,Lima-Perú.

${ }^{3}$ Instituto Peruano de Ingeniería Celular y Manufactura Tisular A.C. . Lima, Perú.

a Médico patólogo.

${ }^{b}$ Estudiante de medicina.

Citar como: Eugenio Palomino, Jesús E. Talavera. Carcinoma indiferenciado con células gigantes de tipo osteoclastos en la cabeza de páncreas. Rev. Fac. Med. Hum. Octubre 2019; 19(4):114-119. DOI 10.25176/RFMH.v19i4.2346 
Las características clínicopatológicas, de imagen y la apariencia tomográfica no están bien definidas ${ }^{5}$. Además, la patogénesis y la histogénesis de estos tumores es muy discutida.

A continuación, presentamos un caso que describe a un paciente con UC-OGCs de cabeza de páncreas.

\section{REPORTE DE CASO}

Describimos el caso de un paciente de sexo masculino de 75 años de edad, procedente de Lima sin antecedentes personales de importancia. Tiene un tiempo de enfermedad de 2 meses y medio, de inicio insidioso y curso progresivo. Presenta dolor abdominal en epigastrio, opresivo, con intensidad progresiva que empeora con la ingesta de alimentos. Días después, el cuadro se exacerba con episodios de melena y pérdida de peso (aprox. 8kg desde el inicio de su enfermedad). Por esa razón, acude a un centro de salud cercano. Es evaluado y en la tomografía axial computarizada (TAC) se evidencia una tumoración pancreática. El paciente es referido al Hospital Nacional Edgardo Rebagliati Martins (HNERM).

Al momento del ingreso, el paciente estaba despierto, pálido, y adelgazado. En la exploración física sólo se evidenció una masa abdominal dura, dolorosa a la palpación, con bordes irregulares en epigastrio, de $15 \times 10 \mathrm{~cm}$ aproximadamente. El resto del examen físico no presentó más alteraciones. En los exámenes auxiliares: hemoglobina 7,0 g/dl (VN: 13-16 g/dl), abastonados 0\%, albumina $2.55 \mathrm{~g} / \mathrm{dL}$ (VN: 3,5 -5 $\mathrm{g} / 100 \mathrm{~mL}$ ), creatinina 1,05 g/dL (VN: 0,5 - 1,3 mg/ $\mathrm{dL}$ ), perfil de coagulación, examen de orina, glucosa, bioquímico dentro de valores normales. Marcadores Inflamatorios: PCR 2,48 $\mathrm{mg} / \mathrm{dL}$, VSG $33 \mathrm{~mm}$. Marcadores tumorales: CA 19-9 en $14 \mathrm{U} / \mathrm{mL}$, CEA 4,3 $\mathrm{ng} / \mathrm{mL}$, AFP 1,8 ng/mL. La endoscopia digestiva alta se describe compresión extrínseca en la curvatura menor y pared anterior de cuerpo gástrico; el píloro se encuentra permeable, a través de él se observa lesión polipoidea que protruye su luz a nivel del bulbo duodenal; en el duodeno se observó una amplia lesión poliploide que ocupa bulbo y primera porción de este ( $5 \mathrm{~cm}$ aprox.), también la presencia de una lesión ulcerada en extremo proximal de superficie regular, friable y blanda a la toma de biopsia; no se identifica base y la mucosa se encuentra eritematosa. En base a la historia clínica y los exámenes realizados, se llega a la conclusión de que el paciente presenta una lesión subepitelial en duodeno, a descartar una tumoración pancreática.

En los estudios de imagen realizados a partir del $3^{\text {er }}$ día de ingreso al servicio de medicina interna fueron: La primera TAC contrastada realizada concluye que los hallazgos tomográficos muestran lesión sugestiva de neoplasia maligna (NM) de la cabeza del páncreas, que impacta y desplaza el antro gástrico, primera y segunda porción del duodeno y probablemente las infiltre, condicionando dilatación del conducto de Wirsung, además de líquido libre subfrénico derecho en poca cantidad (Fig. 1). La tercera TAC realizada 20 días después muestra: Páncreas con lesión sólida quística heterogénea, de $9 \times 5,3 \mathrm{~cm}$, localizada en el cuello y cuerpo de la glándula que comprime el cuerpo gástrico, amplía el marco duodenal que tiene aspecto neoformativo. Sin presencia de líquido libre en cavidad abdominal. Prominencia de la próstata (Fig. 2).

Posteriormente, es sometido a una intervención quirúrgico con el diagnostico preoperatorio de tumor de cabeza de páncreas. Se le realiza una resección en bloque de la tumoración en la cabeza de páncreas, colecistectomía, gastrectomía parcial, duodenectomía, biopsia hepática, y una derivación biliodigestiva en $Y$ de Roux. Los hallazgos fueron los siguientes: Tumor de 10x8x7 cm. a nivel de la cabeza de páncreas que infiltra el duodeno en su primera y parte de su segunda porción, hígado a nivel de borde posterior el segmento III, vasos mesentéricos y vena porta (Fig. 3).

El reporte anatomopatológico concluye que la muestra poseía poblaciones celulares de diferente estirpe (Fig. $4 \mathrm{~A})$, la presencia de células multinucleada de tipo osteoclasto (Fig. 4B), CD68 (+), PACK (+), vimentina (+) CD 34 (-). A la prueba inmunohistoquímica para citoqueratina demostraron evidencia de presencia de población celular maligna (Fig. 4C).

El paciente tuvo una evolución postoperatoria favorable. Con la herida operatoria en buena evolución, buena tolerancia oral, fue dado de alta a los 7 días, posteriormente se le perdió el seguimiento. 


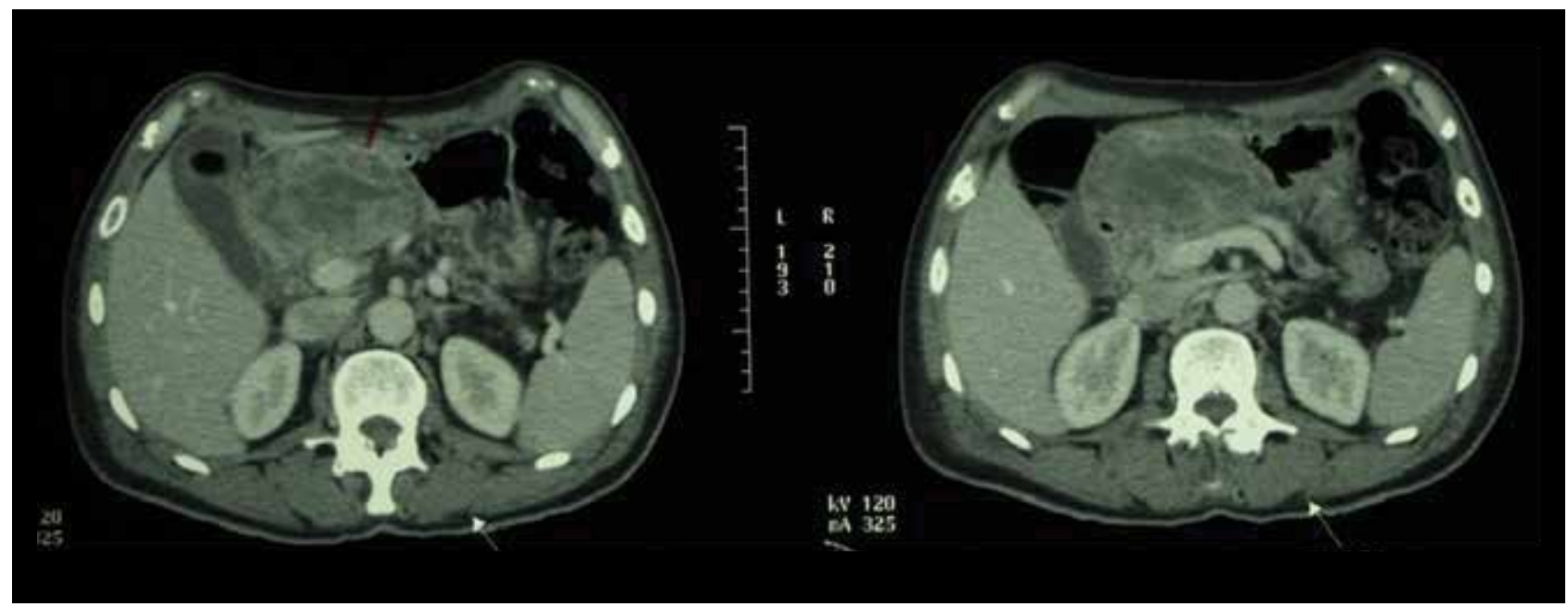

Figura 1. Páncreas con extensa lesión neoformativa sólida dependiente de la cabeza, mide 4,8x7,7cm, en sentido anteroposterior y transversal, tiene bordes parcialmente definidos, contacta con el antro gástrico así como con la primera y segunda porción del duodeno, no existiendo una adecuada diferenciación con estas estructuras probablemente las infiltre, el cuerpo y la cola pancreática son de aspecto atrófico con dilatación del Wirsung.

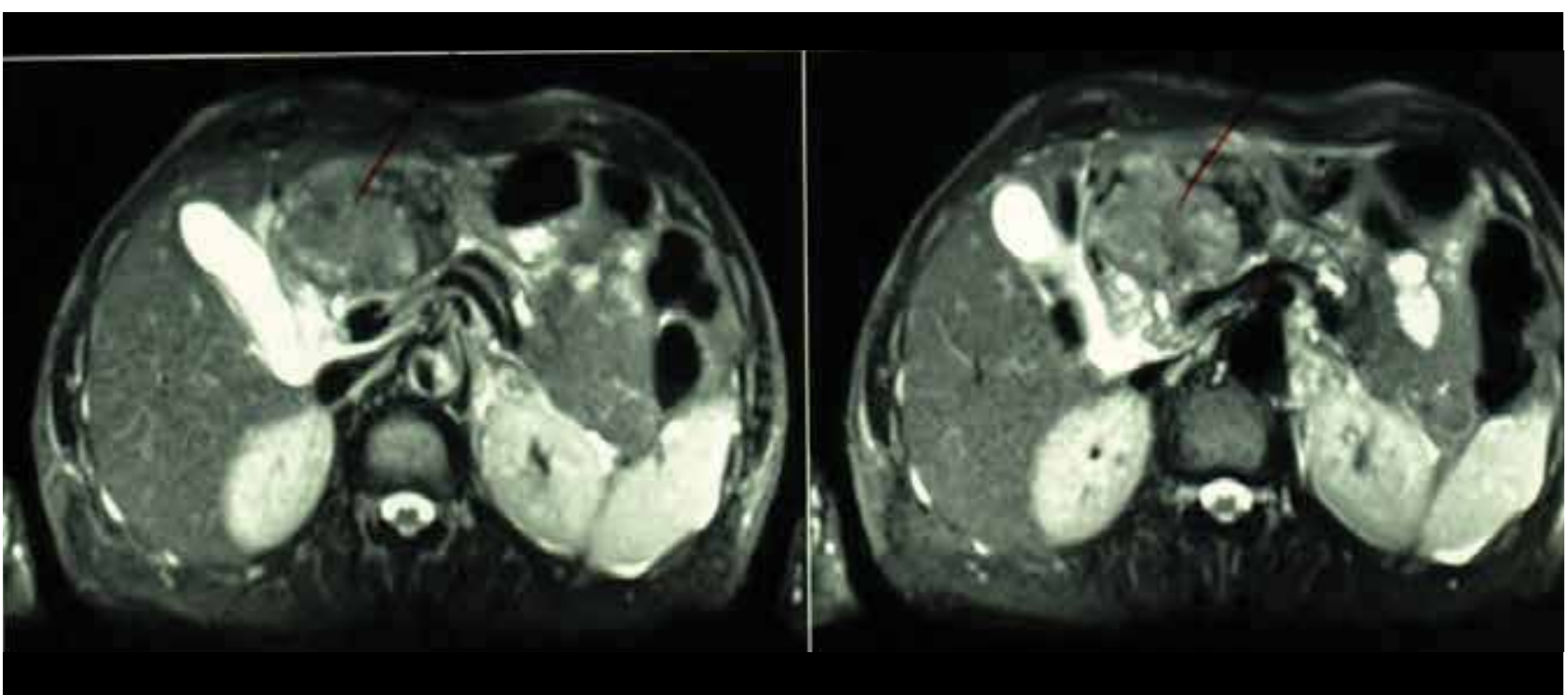

Figura 2. El páncreas muestra una extensa lesión solida quística heterogénea de $9 \times 5,3 \mathrm{~cm}$ localizado en el cuello y cuerpo de la glándula, condiciona dilatación y atrofia distal, comprime el cuerpo gástrico, amplía el marco duodenal, tiene aspecto neoformativo.

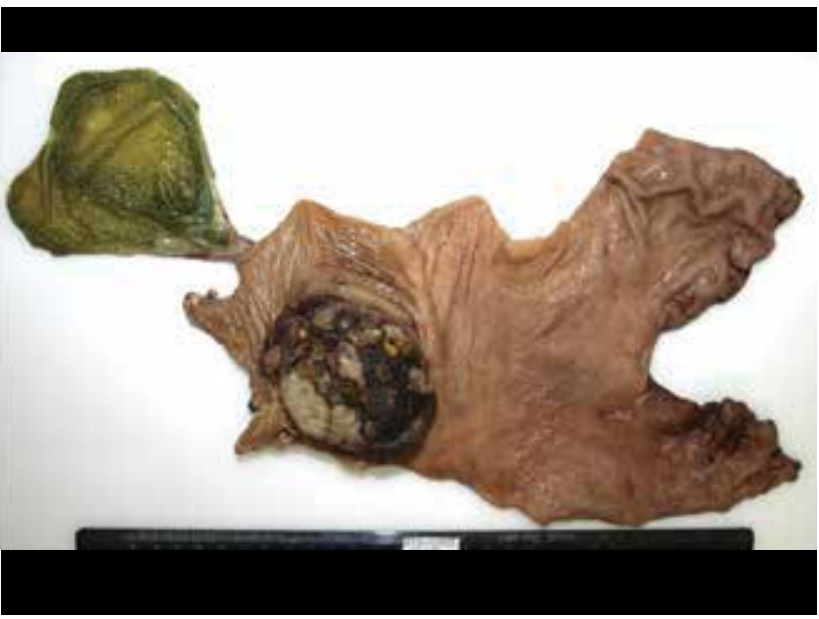

Figura 3. Macrofotografía del espécimen, observándose estómago distal, primero su segunda porción duodenales y vesícula con vía biliar extrahepática. Destaca una neoplasia exofítica, deformando el duodeno y estómago distal, irregularmente ovalada, de $7,5 \mathrm{~cm}$ de diámetro mayor, con áreas de color pardusco, negro y grisáceo. 


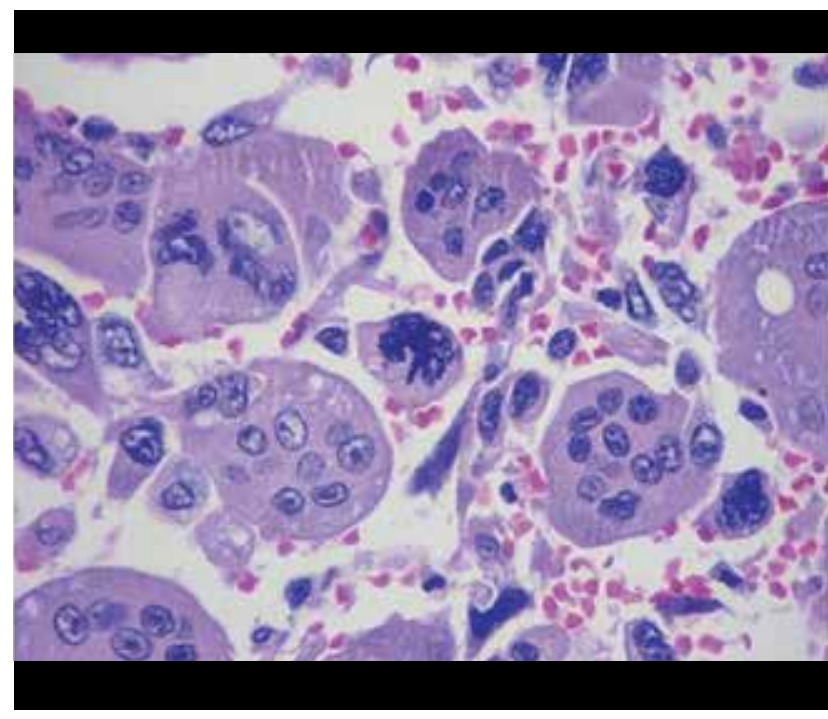

Figura 4-A. Se observa dos poblaciones celulares distintas: las células del carcinoma indiferenciado, con marcada anaplasia y mitosis atípicas. La otra población corresponde a las células gigantes multinucleadas osteoclastoides, de naturaleza benigna, reactiva. (Coloración H-E, 400 X).

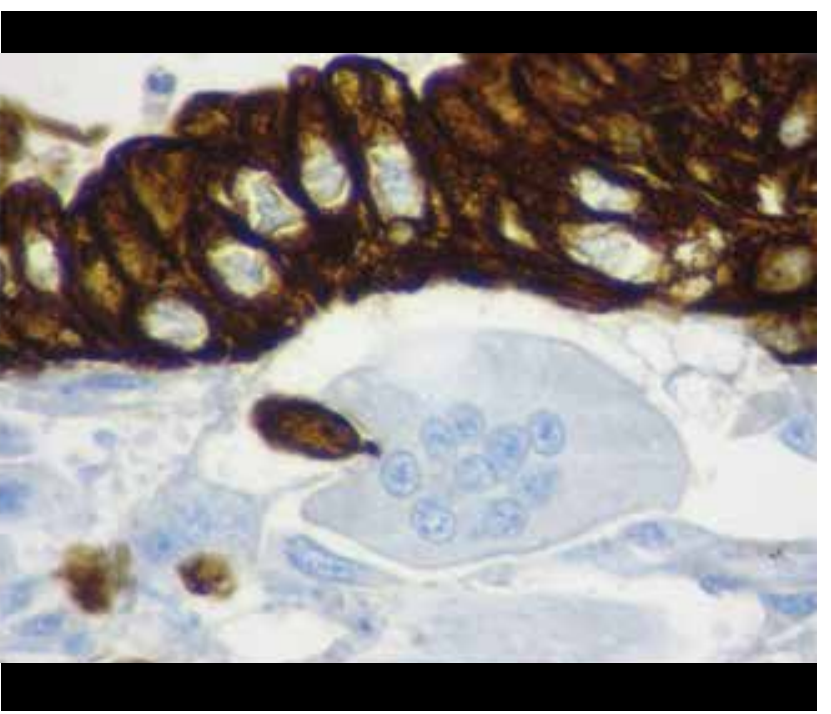

Figura 4-C. Prueba de inmunohistoquímica para Citoqueratina, demostrando la naturaleza epitelial de la población célular maligna. La célula gigante multinucleada es negativa. (Coloración H-E, 400 X).

\section{DISCUSIÓN}

Nuestro paciente un adulto mayor de 75 años de edad, presentaba un tiempo de enfermedad considerable sin presencia de sintomatología relevante hasta que el tumor estuvo en etapas avanzadas, debutando con molestias gástricas someras, pasando a la pérdida de peso. En los exámenes diagnósticos realizados sugirieron la presencia de un tumor que comprometía el estómago, duodeno y páncreas. $Y$ el estudio anatomopatológico posterior dio el

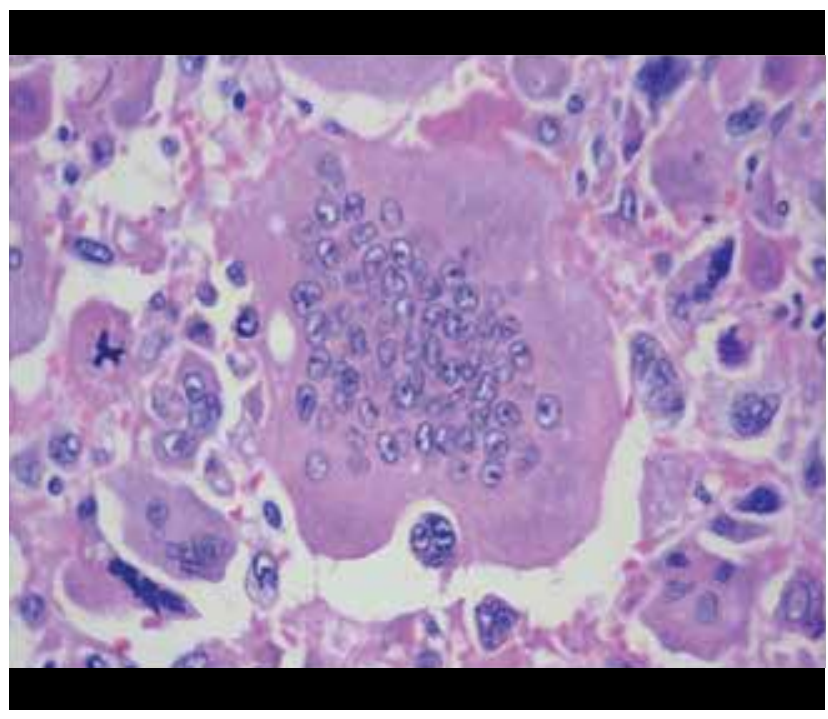

Figura 4-B. Célula gigante multinucleada osteoclastoide fagocitando una células neoplásica del carcinoma indiferenciado, con 3 núcleos pleomórficos y pérdida de la relación núcleo/ citoplasma. (Coloración H-E, 400 X).

diagnóstico definitivo de esta patología. El Carcinoma indiferenciado con células gigantes tipo osteoclasto de páncreas, es una neoplasia muy poco frecuente. Se encuentra catalogada en la clasificación de tumores pancreáticos por la Organización Mundial de la Salud en su edición del año $2010^{6}$.

Esta patología se caracteriza por la presencia de dos poblaciones celulares: células epiteliales mononucleares polimórficas indiferenciadas y células gigantes multinucleadas benignas. El compromiso ganglionar y las metástasis son inusuales, sin embargo, la neoplasia suele invadir estructuras adyacentes. Se ha descrito que la sobrevida promedio en estos casos es de 11 meses $^{7}$.

Se cuenta con pocos casos en la literatura y no sea encontrado ningún caso reportado en Latinoamérica. La prevalencia hombre y mujer es de 2:3 y la edad promedio es 67 años con un rango de 33 a 88 años $^{8}$. Este tipo de tumor puede localizarse en diferentes órganos, en el sistema digestivo su localización más frecuente es la vesícula biliar y el páncreas ${ }^{8}$. En nuestro paciente los estudios por imagen sugerían la presencia del tumor localizado en la cabeza del páncreas con extensión hasta la muscularis mucosae del duodeno adyacente, cuyo diagnóstico inicial fue retrasado debido a la presentación atípica del cuadro clínico y la escasez de casos similares.

La presentación clínica de esta patología generalmente se asocia a dolor abdominal, masa palpable, pérdida 
de peso, fatiga, anorexia e ictericia9. Comúnmente localizado en el cuerpo y la cola del páncreas ${ }^{4}$, en nuestro caso el tumor estaba localizado en la cabeza del páncreas. El diagnóstico diferencial de esta patología incluye lesiones quísticas, como los carcinomas pancreáticos ductales o tumores neuroendocrinos ${ }^{10}$. En nuestro paciente los hallazgos clínicos de mayor importancia fueron el dolor abdominal y la pérdida de peso. No se cuenta actualmente con información específica sobre el curso clínico o pautas para el manejo de esta patología. Incluso el tratamiento adyuvante con quimioterapia no está completamente establecido ${ }^{4}$, sin embargo la radioterapia ha sido aplicada como base a la radio sensibilidad de los tumores de células gigantes de los huesos a está.

El origen histológico de UC-OGC es actualmente un tema de controversia pues algunos postulados, mediante técnicas de microscopia electrónica, indican que se origina de tejidos mesenquimales ${ }^{11}$. Otros autores sugieren que dicho tumor se origina de un precursor de células pluripotenciales las cuales tienen la capacidad de diferenciarse en fenotipos específicos ${ }^{4}$. En nuestro paciente el informe anátomo patológico confirmó en efecto la presencia de componente de células gigantes tipo osteoclastos y componente sarcomatoide fusocelulares.

En el presente caso el paciente ya presentaba factores de riesgo ya mencionados anteriormente, sin embargo, la sintomatología inicial es muy inespecífica, haciendo que en único medio actual para el diagnóstico sea el estudio anátomo patológico. Actualmente, las pautas sobre el manejo y pronóstico no están claro, sin embargo se reconoce un pronóstico pobre con la muerte del paciente a los pocos meses del diagnóstico ${ }^{12}$. Agregado a ello, se identifican algunos factores de mal pronóstico como edad avanzada, sexo masculino, tumores pequeños, metástasis positiva y un componente concomitante de adenocarcinoma ductal ${ }^{13}$. Nuestro paciente cumplía con algunos de los factores de mal pronóstico previamente mencionados, después de la resección quirúrgica del tumor la paciente, posterior a la cirugía se le perdió el seguimiento; sin embargo la literatura describe que este tipo de tumor tiene una considerable taza de recurrencias ${ }^{14}$. Posteriormente el paciente esta con vida, y por el momento no muestran indicios de recurrencia del tumor después de ser sometido a la cirugía. Lo cual sugiere que la pancreatoduodenectomía es el tratamiento que mejor resultados ofrece para el tratamiento de este tipo de tumor. No hay quimioterapia estándar, sin embargo, la mayoría de reportes de caso han usado gemcitabina como terapia de mantenimiento. Por lo cual es necesario estudiar más casos similares, compararlos, continuar con un seguimiento a largo plazo y evaluar la efectividad de la pancreatoduodenectomía en este tipo de pacientes para así obtener más datos sobre el curso clínico de la enfermedad, guías sobre su manejo y datos más precisos sobre su tratamiento y pronostico en estos pacientes.

\section{CONCLUSIÓN}

El carcinoma indiferenciado con células gigantes de tipo osteoclastos es un tipo raro de tumor y debido a ello, no se dispone de datos clínicos suficientes para un tratamiento que garantice una mayor tasa de supervivencia en los pacientes, sin embargo, la pancreatoduodenectomía es actualmente la alternativa que mejores resultados ofrece, por otra parte, el pronóstico después de la cirugía y la tasa de recurrencia sigue sin estar claro.

Contribuciones de autoría: Los autores participaron en la generación, recolección de información, redacción y versión final del artículo original.

Financiamiento: Autofinanciado.

Conflicto de interés: Los autores declaran no tener conflictos de interés en la publicación de este artículo.

Recibido: 20 de julio del 2019

Aprobado: 07 de septiembre del 2019

Correspondencia: Jesús E. Talavera

Dirección: Jr. Monte Cedro 413, Santiago de surco. Lima, Perú.

Teléfono: (511) 959706046

Correo:enrique7.talav@gmail.com 


\section{REFERENCIAS BIBLIOGRÁFICAS}

1. Tratamiento del cáncer de páncreas $\left(\mathrm{PDQ}^{\circledR}\right)$-Versión para profesionales de salud [Internet]. Instituto Nacional del Cáncer. 2019 [citado 2 de septiembre de 2019]. Disponible en: https://www.cancer.gov/espanol/ tipos/pancreas/pro/tratamiento-pancreas-pdq.

2. Molberg KH, Heffess C, Delgado R, Albores-Saavedra J. Undifferentiated carcinoma with osteoclast-like giant cells of the pancreas and periampullary region. Cancer. abril de 1998;82(7):1279-87. Disponible en: https://doi.org/10.1002/(SICl)1097-0142(19980401)82:7<1279::AIDCNCR10>3.0.CO;2-3

3. Rosai J. Carcinoma of pancreas simulating giant cell tumor of bone. Electron-microscopic evidence of its acinar cell origin. Cancer. agosto de 1968;22(2):333-44. Disponible en: https://doi.org/10.1002/10970142(196808)22:2<333::AID-CNCR2820220210>3.0.CO;2-A

4. Georgiou GK, Balasi E, Siozopoulou V, Tsili A, Fatouros M, Glantzounis G. Undifferentiated carcinoma of the head of pancreas with osteoclastlike giant cells presenting as a symptomatic cystic mass, following acute pancreatitis: Case report and review of the literature. Int J Surg Case Rep. 22 de diciembre de 2015;19:106-8. Disponible en: https://doi. org/10.1016/j.ijscr.2015.12.023

5. Bauditz J, Rudolph B, Wermke W. Osteoclast-like giant cell tumors of the pancreas and liver. World J Gastroenterol. diciembre de 2006;12(48):787883. doi: 10.3748 / wjg.v12.i48.7878

6. Chopra S, Wu ML-C, Imagawa DK, Lee J, Gu M. Endoscopic ultrasoundguided fine-needle aspiration of undifferentiated carcinoma with osteoclast-like giant cells of the pancreas: A report of 2 cases with literature review. Diagn Cytopathol. 2007;35(9):601-606. Disponible en https://doi.org/10.1002/dc.20711

7. Singhal A, Shrago SS, Li S-F, Huang Y, Kohli V. Giant cell tumor of the pancreas: a pathological diagnosis with poor prognosis. Hepatobiliary Pancreat Dis Int HBPD INT. agosto de 2010;9(4):433-7. Disponible en: https://europepmc.org/abstract/med/20688610
8. Hickey PT, Nair R, Loven V, Brodsky J, Shah $H$. Undifferentiated carcinoma with osteoclast-like giant cells of the pancreatic tail. Gastroenterology. 2015;148(4):S939. Disponible en: https://kundoc.com/ pdf-tu1936-undifferentiated-carcinoma-with-osteoclast-like-giant-cellsof-the-pancre.html

9. Hur YH, Kim HH, Seoung JS, Seo KW, Kim JW, Jeong YY, et al Undifferentiated carcinoma of the pancreas with osteoclast-like giant cells. J Korean Surg Soc. 1 de agosto de 2011;81(2):146-50. Disponible en: https://doi.org/10.4174/jkss.2011.81.2.146

10. Oehler U, Jürs $M$, Klöppel G, Helpap B. Osteoclast-like giant cel tumour of the pancreas presenting as a pseudocyst-like lesion. Virchows Arch. 1997;431(3):215-218. Disponible en: https://doi.org/10.1007/ s004280050091

11. Kawamoto $Y$, Ome $Y$, Terada K, Hashida K, Kawamoto K, Ito T. Undifferentiated carcinoma with osteoclast-like giant cells of the ampullary region: Short term survival after pancreaticoduodenectomy. Int J Surg Case Rep. 1 de enero de 2016;24:199-202. Disponible en: https://doi.org/10.1016/j.ijscr.2016.04.057

12. Goldberg RD, Michelassi F, Montag AG. Osteoclast-like giant cell tumor of the pancreas: immunophenotypic similarity to giant cell tumor of bone. Hum Pathol. 1991;22(6):618-622. Disponible en: https://doi. org/10.1016/0046-8177(91)90243-I

13. Kobayashi S, Nakano H, Ooike N, Oohashi M, Koizumi S, Otsubo T. Long-term survivor of a resected undifferentiated pancreatic carcinoma with osteoclast-like giant cells who underwent a second curative resection: A case report and review of the literature. Oncol Lett. octubre de 2014;8(4):1499-504. Disponible en: https://doi.org/10.3892/ol.2014.2325

14. Holland R, van Haelst UJGM. Mammary carcinoma with osteoclastlike giant cells: Additional observations on six cases. Cancer. 1 de mayo de 1984;53(9):1963-73. Disponible en: https://doi.org/10.1002/10970142(19840501)53:9<1963::AID-CNCR2820530927>3.0.CO;2-N

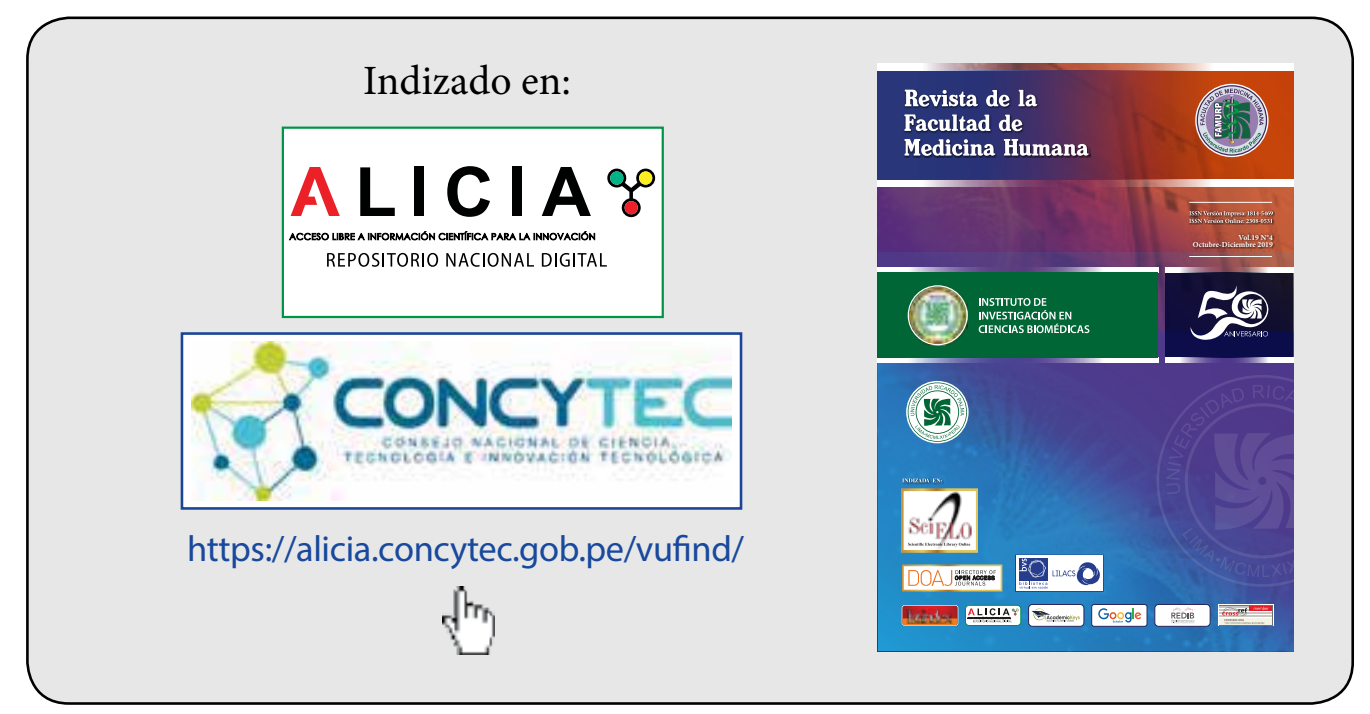

